\title{
ÍNDICE DE GRAVIDADE DA DOENCGA RENAL, INDICADORES ASSISTENCIAIS E MORTALIDADE EM PACIENTES EM HEMODIÁLISE
}

\author{
Cássia Morsch*, Luiz Felipe Gonçalves, Elvino Barros
}

Trabalho realizado na Universidade Federal do Rio Grande do Sul - Faculdade de Medicina - Programa de Pós-Graduação em Ciências Médicas: Nefrologia - Serviço de Nefrologia - Hospital de Clínicas de Porto Alegre, RS.

\section{*Correspondência:}

Rua Machado de Assis, 612,

Porto Alegre, RS,

Cep 90620-260

\begin{abstract}
RESUMO
Oвjetivo. Verificar a associaçãao das comorbidades, quantificadas por meio do índice de gravidade da doença renal (IGDR), com os indicadores assistenciais Kt $N$, hematócrito e albumina sérica, e com mortalidade.

Métodos. Quarenta pacientes renais crônicos em hemodiálise foram acompanhados por 12 meses e avaliados quanto a características sociodemográficas, tempo em diálise, presença de diabetes mellitus, indicadores assistenciais e comorbidades. A influência das comorbidades foi avaliada por meio do IGDR.

Resultados. O IGDR médio dos sobreviventes (85\%) e óbitos (I5\%) foi 22 \pm I4,8 vs $44 \pm 12,4$ ( $p<0,001$ ) e entre pacientes

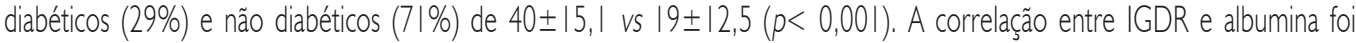
$r=-0,475(p<0,005)$. A maioria dos pacientes com albumina $=3,6 \mathrm{mg} /(82 \%)$ era composta de não diabéticos $(p=0,021)$. Houve correlação do hematócrito com a albumina, sendo $r=0,544,(p<0,00 \mathrm{I})$. $\mathrm{OKt} N \mathrm{~N}$ não teve associação com outras varíáveis. A razão de chance de óbito para cada ponto do IGDR foi de $10 \%(p=0,0093)$.
\end{abstract}

ConcLusÃo. O IGDR é um bom instrumento para avaliar comorbidade em pacientes em hemodiálise, sendo confiável para comparar grupos de pacientes e predizer mortalidade.

UnIteRmos: Hemodiálise. Morbidade. Mortalidade. Diabetes mellitus.

\section{INTRODUÇÃo}

A gravidade de uma doença está relacionada ao grau de distúrbios fisiológicos ou morfológicos de um paciente com determinada enfermidade. Medidas de gravidade são necessárias para descrever e comparar pacientes de diferentes unidades, assim como avaliar e prever a qualidade dos cuidados. Entretanto, existem poucos instrumentos que ajustam os resultados para gravidade da comorbidade e poucos têm sido testados na populaçãao de pacientes com insuficiência renal crônica'. Alguns destes instrumentos são o índice de Karnofsky², que avalia a capacidade funcional, o índice de doenças coexistentes $^{3}$ e $o$ índice de gravidade da doença renal ${ }^{4}$.

$O$ índice de gravidade da doença renal - IGDR (The endstage renal disease severity index - ESRD-SI), instrumento proposto por Craven $^{4}$, foi desenvolvido para avaliar a influência dos aspectos comórbidos na evolução dos pacientes renais crônicos. Este instrumento é de fácil aplicabilidade, podendo ser utilizado rotineiramente na prática clínica ${ }^{5}$.

Inúmeros indicadores têm sido utilizados para avaliar a qualidade assistencial oferecida aos pacientes em hemodiálise. Entre eles, o Kt/ $V$ - como marcador de adequação dialítica ${ }^{6-9}$, o hematócrito - como marcador de anemia 10,11 e a albumina - como marcador de estado nutricional $\left.\right|^{12,13,14}$. Esses indicadores são os mais estudados, e estão diretamente relacionados à morbi-mortalidade destes pacientes.

O propósito deste estudo foi avaliar o IGDR e alguns indicadores assistenciais como marcadores de mortalidade em pacientes renais crônicos em tratamento hemodialítico.

\section{Métodos}

Foi realizado um estudo de coorte, prospectivo, não controlado e contemporâneo para avaliar a associação do IGDR e alguns indicadores assistenciais com mortalidade de pacientes em hemodiálise, na Unidade de Hemodiálise do Serviço de Nefrologia do Hospital de Clínicas de Porto Alegre, RS, Brasil.

O trabalho foi elaborado de acordo com as diretrizes e normas regulamentadoras de pesquisas envolvendo seres humanos, tendo sido avaliado e aprovado pela Comissão de Pesquisa e Ética em Saúde da instituiçãa.

Participaram do estudo 40 pacientes com insuficiência renal terminal de diversas etiologias. Foram incluídos pacientes de ambos os sexos, adultos, com idade igual ou superior a 18 anos. Além disso, necessitavam estar no programa de hemodiálise há mais de três meses, sem interrupção, apresentando condições plenas de expressar-se e informar, corretamente, seus problemas e estado de saúde. Finalmente, para participar do estudo era necessária a assinatura espontânea do consentimento informado.

Não foram admitidos no estudo os pacientes portadores de doença neoplásica conhecida, pacientes que rejeitaram transplante renal nos últimos seis meses ou trocaram de modalidade de diálise nos últimos três meses e os pacientes que se recusaram a assinar o termo de consentimento.

\section{Avaliação da morbidade}

Para avaliar os aspectos comórbidos foi utilizado o IGDR, medida na qual estão incluídas dez categorias de doenças comuns em urêmicos: 
cardiovascular, cerebrovascular, doença vascular periférica, neuropatia periférica, doença óssea, doença respiratória, déficit visual, neuropatia autonômica e doença gastrointestinal, acesso e ocorrências dialíticas, diabetes e uma categoria aberta ${ }^{4}$.

Cada categoria apresenta uma variação de gravidade com uma graduação escolhida, conforme o seguinte critério:

। - $3 \Rightarrow$ para alterações leves ou bioquímicas de doença;

$4-6 \Rightarrow$ para sinais físicos moderados a intensos, com implicações de prognóstico ou determinando algum tipo de prejuízo;

$7-8 \Rightarrow$ para uma alteração física debilitante ou de prognóstico ruim;

9 - $10 \Rightarrow$ para uma condição potencialmente fatal.

Cada categoria recebe um escore, e o IGDR é a soma total dos escores.

Os instrumentos foram preenchidos por médicos nefrologistas responsáveis pelos pacientes em hemodiálise. Cada paciente recebeu seu escore no início do seguimento.

Os pontos de corte para se considerar o grau de comorbidade do IGDR foram:

- de 0 a 10 - baixo risco;

- de Il a 30 - médio risco;

- > 30 - alto risco.

\section{Avaliação de indicadores assistenciais}

A adequação da dose de diálise foi medida por meio do método de cinética da uréia, empregando o modelo monocompartimental de volume variável. $\mathrm{O}$ alvo estabelecido neste estudo foi Kt $N$ mínimo de $1,2^{15}$. Para a avaliação do grau de anemia dos pacientes, foi utilizado o hematócrito, tendo como alvo estabelecido valores mínimos de $33 \% 15,16$. Como marcador do estado nutricional foi utilizada a albumina (As), avaliada em três momentos durante o seguimento. $\mathrm{O}$ alvo estabelecido neste estudo foi $\mathrm{As}=3,6 \mathrm{~g} / \mathrm{d}^{\llcorner} \mathrm{L}^{13,14}$.

Essas avaliações foram realizadas como parte da rotina de acompanhamento dos pacientes e aconteceram nas diálises do meio da semana, nas segundas semanas de cada mês. Os pontos de corte nos indicadores assistenciais (alvos estabelecidos neste estudo) foram baseados em recomendações internacionais de qualidade assistencial em nefrologia $10,1,12,16$. Os pacientes com diagnóstico de diabetes mellitus como fator etiológico da insuficiência renal crônica foram avaliados separadamente com o propósito de verificar o impacto do diabetes nos resultados de adequação dialítica $(\mathrm{Kt} N \mathrm{~N})$, hematócrito, albumina sérica e mortalidade. Também foi verificada a magnitude da interação entre diabetes mellitus e o IGDR para predizer mortalidade, ou seja, a importância das comorbidades em diabéticos e não diabéticos como fator preditivo para o evento óbito.

\section{Análise estatística}

Para cada paciente foram utilizadas as médias dos resultados de Kt/ $V$, hematócrito e albumina sérica obtidos durante o seguimento. A associação entre mortalidade, características sociodemográficas, diabetes mellitus e indicadores assistenciais foi avaliada por meio do teste exato de Fisher, teste te Mann-Whiney para variáveis contínuas sem distribuição normal (tempo de hemodiálise). A magnitude da associaçãao entre diabetes mellitus e mortalidade foi apresentada com a razão de chances e IC de $95 \%$.

As diferenças de magnitude do grau de morbidade (IGDR) em relaçãa às variáveis sociodemográficas (mensuradas de forma qualitativa), de mortalidade e etiologia da insuficiência renal crônica (diabetes mellitus vs nãodiabetes mellitus), foi avaliada por meio do teste t de Student. A razão de chances entre indicadores assistenciais, diabetes mellitus, IGDR e mortalidade, com IC 95\%, foi calculada por meio da regressão logística. A interação entre diabetes mellitus e IGDR foi avaliada através do teste Qui quadrado de Wald, por meio de regressão logística múltipla. A avaliaçãa da correlação entre o IGDR e os indicadores assistenciais foi verificada por meio do coeficiente de correlação de Pearson. Foram considerados significativos os valores de $p$ menores que 0,05 .

\section{Resultados}

A média de idade dos pacientes foi de 50,5 17 anos, sendo o mais jovem de 18 anos, e o mais idoso de 95 anos. Trinta e sete por cento dos pacientes apresentavam idade superior a 60 anos. A maioria dos pacientes era constituída de homens (67,5\%), caucasóides (72,5\%), casados (70\%), que estavam em tratamento em hemodiálise há mais de um ano (70\%). Apenas 12,5\% deste grupo desempenhavam alguma atividade laborativa formal remunerada e, em relação à escolaridade, metade da amostra estudada tinha formação inferior ao primeiro grau completo.

As principais causas do desenvolvimento da insuficiência renal crônica nesses pacientes, à semelhança de outros países, foram diabetes mellitus, observado em $29 \%$ do total de pacientes, seguido de hipertensão arterial sistêmica (23\%), glomerulopatias (13\%) e de doença renal policística (10\%). No período de seguimento (I 2 meses), seis (15\%) pacientes foram a óbito e três $(7,5 \%)$ foram submetidos a transplante renal.

Durante o período de acompanhamento não foi observada diferença significativa entre as variáveis sócio-demográficas e tempo em diálise dos sobreviventes, comparados àqueles que foram a óbito, conforme demonstrado nas Tabelas I e 2. Os fatores de maior impacto sobre a mortalidade foram a presença de diabetes mellitus e o IGDR. A média geral dos escores de gravidade da doença renal foi de 25 , sendo que as doenças cardiovasculares, como seria de se esperar, foram as que mais contribuíram para compor este índice $(3,9 \pm 3,5)$, seguidas de doença óssea $(3,7 \pm 2,2)$, doença vascular periférica $(3,0 \pm 2,7)$ e acesso vascular e diálise $(2,5 \pm 2,9)$. Entre os pacientes estudados, 33\% apresentaram escores superiores a $31,49 \%$ entre II e 30 e 18\% apresentaram escores inferiores a 10.

A análise de regressão logística simples, realizada com o índice de gravidade da doença renal mostrou, neste estudo, haver uma razão de chances de mortalidade de I, 10 (IC 95\%: I,02-I, I8) para cada ponto acrescido no IGDR $(p=0,0093)$. A avaliação geral dos escores de IGDR mostrou que a média dos pacientes sobreviventes foi significativamente menor do que a dos pacientes que foram a óbito (Tabela 2). Esse mesmo escore também foi menor nos pacientes não diabéticos. Os escores dos sobreviventes não diabéticos foram significativamente menores que os escores dos pacientes não diabéticos que foram a óbito 
Morsch C et al.

\begin{tabular}{|c|c|c|c|c|c|c|}
\hline & \multicolumn{5}{|c|}{ Tabela I - Avaliação sociodemográfica e de tempo em diálise entre pacientes vivos e óbitos } & \\
\hline \multirow{2}{*}{ Características } & & \multicolumn{2}{|c|}{ Óbito } & \multicolumn{2}{|c|}{ Vivos } & \multirow{2}{*}{$p^{*}$} \\
\hline & & $\mathrm{n}$ & $\%$ & $\mathrm{n}$ & $\%$ & \\
\hline Sexo & $\begin{array}{l}\text { Masculino }(n=27) \\
\text { Feminino }(n=13)\end{array}$ & $\begin{array}{l}4 \\
2\end{array}$ & $\begin{array}{l}15 \\
15\end{array}$ & $\begin{array}{l}23 \\
11\end{array}$ & $\begin{array}{l}85 \\
85\end{array}$ & 1,000 \\
\hline Cor & $\begin{array}{c}\operatorname{Branca}(n=29) \\
\text { Nãobranca }(n=\mid 1)\end{array}$ & $\begin{array}{l}6 \\
0\end{array}$ & $\begin{array}{l}21 \\
0\end{array}$ & $\begin{array}{l}23 \\
11\end{array}$ & $\begin{array}{l}79 \\
100\end{array}$ & 0,162 \\
\hline Escolaridade & $\begin{array}{l}I^{0} \text { grau inc }(n=20) \\
\text { I }^{\circ} \text { grau ou }+(n=20)\end{array}$ & $\begin{array}{l}3 \\
3\end{array}$ & $\begin{array}{l}15 \\
15\end{array}$ & $\begin{array}{l}17 \\
17\end{array}$ & $\begin{array}{l}85 \\
85\end{array}$ & 1,000 \\
\hline Estado civil & $\begin{array}{c}\text { Casado }(n=28) \\
\text { Não casado }(n=12)\end{array}$ & $\begin{array}{l}3 \\
3\end{array}$ & $\begin{array}{l}11 \\
25\end{array}$ & $\begin{array}{c}25 \\
9\end{array}$ & $\begin{array}{l}90 \\
75\end{array}$ & 0,341 \\
\hline Ocupação & $\begin{array}{c}\text { Não empregado }(n=35) \\
\text { Empregado }(n=5)\end{array}$ & $\begin{array}{l}4 \\
2\end{array}$ & $\begin{array}{l}11 \\
40\end{array}$ & $\begin{array}{c}31 \\
3\end{array}$ & $\begin{array}{l}89 \\
60\end{array}$ & 0,154 \\
\hline Tempo HD & $\begin{array}{l}3 \mathrm{~m} \text { a I ano }(n=12) \\
\text { mais de I ano }(n=28)\end{array}$ & $\begin{array}{l}0 \\
6\end{array}$ & $\begin{array}{c}0 \\
21\end{array}$ & $\begin{array}{l}12 \\
22\end{array}$ & $\begin{array}{l}100 \\
79\end{array}$ & 0,153 \\
\hline Idade & $\begin{array}{l}18 \text { a } 59 \text { anos }(n=25) \\
60 \text { a } 95 \text { anos }(n=15)\end{array}$ & $\begin{array}{l}2 \\
4\end{array}$ & $\begin{array}{l}8 \\
27\end{array}$ & $\begin{array}{l}23 \\
11\end{array}$ & $\begin{array}{l}92 \\
73\end{array}$ & 0,174 \\
\hline
\end{tabular}

\begin{tabular}{|c|c|c|c|}
\hline \multicolumn{4}{|c|}{$\begin{array}{l}\text { Tabela } 2 \text { - Médias dos escores de IGDR, idade e } \\
\text { tempo em hemodiálise em relação à mortalidade }\end{array}$} \\
\hline $\begin{array}{l}\text { Óbitos } \\
\mathrm{n}(\%)\end{array}$ & $\begin{array}{c}\text { IGDR } \\
* \text { (média } \pm \text { dp) }\end{array}$ & $\begin{array}{c}\text { Idade } \\
\text { (anos) } \\
\text { *(média } \pm d p)\end{array}$ & $\begin{array}{c}\text { Tempo em } \\
\text { hemodiálise (meses) } \\
\text { **(mediana) }\end{array}$ \\
\hline $\begin{array}{l}\text { Sim } \\
6(15 \%)\end{array}$ & $44 \pm 12,4$ & $59 \pm \overline{24}, 4$ & 33 \\
\hline $\begin{array}{l}\text { Não } \\
34(85 \%) \\
p\end{array}$ & $\begin{array}{l}22 \pm \mid 4,8 \\
<0,00 \mid\end{array}$ & $\begin{array}{c}50,3 \pm 14,6 \\
0,239\end{array}$ & $\begin{array}{c}19 \\
0,231\end{array}$ \\
\hline
\end{tabular}

\begin{tabular}{|c|c|c|c|c|}
\hline \multicolumn{5}{|c|}{$\begin{array}{l}\text { Tabela } 3 \text { - Médias dos escores de IGDR entre } \\
\text { diabéticos e não diabéticos associados à mortalidade }\end{array}$} \\
\hline \multirow{2}{*}{\multicolumn{2}{|c|}{$\begin{array}{c}\text { Diabéticos } \\
=12(29 \%) \\
(\text { média } \pm d p) \\
40 \pm 15,1\end{array}$}} & \multirow{2}{*}{\multicolumn{2}{|c|}{$\begin{array}{c}\text { Não diabéticos } \\
N=28(71 \%) \\
(\text { média } \pm \text { dp }) \\
19 \pm 12,5\end{array}$}} & $p$ \\
\hline & & & & $<0,00 \mid$ \\
\hline Sobreviventes & Óbitos & Sobreviventes & & tos \\
\hline $40 \pm 13,4$ & $\begin{array}{l}47 \pm 14,5 \\
p=0,446\end{array}$ & $17,6 \pm 11,6$ & & \\
\hline
\end{tabular}

Tabela 4 - Avaliação dos indicadores assistenciais entre pacientes vivos e que foram a óbito

\begin{tabular}{|c|c|c|c|}
\hline & $\mathrm{Kt} / \mathrm{V}$ & Hematócrito & Albumina \\
\hline Óbitos $(n=6)$ & $\overline{1,27 \pm 0,13}$ & $32,65 \pm 5,1$ & $3,28 \pm 0,77$ \\
\hline $\operatorname{Vivos}(n=34)$ & $1,28 \pm 0,18$ & $33,63 \pm 2,3$ & $3,7 \pm 0,24$ \\
\hline$p^{*}$ & 0,883 & 0,659 & 0,231 \\
\hline
\end{tabular}

*Teste T de Student $(p=0,0 \mid 6)$. Não houve diferença nos escores de IGDR entre os diabéticos sobreviventes e os que foram a óbito (Tabela 3).

A mortalidade entre os diabéticos, durante o período de seguimento, foi de $33 \%$ vs $7 \%$ entre os não diabéticos. É importante salientar que o risco estimado de pacientes diabéticos para o evento óbito, em relação aos pacientes não diabéticos foi de 4,7 (IC 95\%, $0,984$ a 22, I38; $p=0,055)$. O modelo de regressão logística aplicado para avaliar o impacto na mortalidade demonstrou que, entre as variáveis independentes diabetes mellitus e IGDR, somente o IGDR apresentou significância estatística (IC 95\%: I,002 - I, I 85, p = 0,045). A interação entre diabetes mellitus e IGDR não foi significativa $(p=0,29)$.

As variáveis sociodemográficas não influenciaram o IGDR, embora os pacientes com menos tempo de hemodiálise (abaixo de um ano), quando a amostra foi estratificada em três níveis, apresentaram uma tendência a IGDR menor do que aqueles com maior tempo de hemodiálise $(p=0,060)$.

Durante o período de seguimento, $63 \%$ dos resultados de Kt $N$ dos pacientes foram iguais ou superiores a I,2. Em relação ao hematócrito, $68 \%$ dos resultados dos pacientes foram iguais ou superiores a $33 \%$, e $70 \%$ dos resultados referentes à albumina foram iguais ou superiores a 3,6\%/dL.

A avaliação dos indicadores assistenciais $\mathrm{Kt} N$, hematócrito e albumina dos pacientes que foram a óbito, comparados com os dos pacientes sobreviventes, mostrou não haver diferenças significativas entre esses dois grupos (Tabela 4). Estes mesmos indicadores também não foram diferentes em pacientes com diagnóstico de diabetes mellitus em relaçãao aos não diabéticos. No entanto, em relaçãa à albumina, 58\% dos pacientes diabéticos tiveram resultados inferiores ao alvo, enquanto que somente $18 \%$ dos não diabéticos não atingiram o alvo estabelecido $(p=0,021$ - Tabela 5).

Os diferentes indicadores assistenciais também foram testados para avaliar uma possível correlação entre eles e o IGDR. Observou-se uma correlação negativa entre albumina e IGDR $(r=-0,475 ; p=0,002)$, assim como uma correlação positiva significativa entre a albumina e o hematócrito $(r=0,544 ; p<0,00 \mathrm{I})$. 


\begin{tabular}{|c|c|c|c|c|c|c|c|c|c|c|}
\hline \multirow[t]{2}{*}{ Indicador } & \multicolumn{2}{|c|}{ Óbitos } & \multicolumn{3}{|c|}{ Sobreviventes } & \multicolumn{2}{|c|}{ Diabéticos } & \multicolumn{3}{|c|}{ Não Diabéticos } \\
\hline & $\mathrm{n}$ & $\%$ & $\mathrm{n}$ & $\%$ & $p^{*}$ & $n$ & $\%$ & $n$ & $\%$ & $p^{*}$ \\
\hline $\mathrm{Kt} / \mathrm{V}<\mathrm{I}, 2$ & 2 & $\overline{13}$ & $\overline{13}$ & $\overline{87}$ & & $\overline{4}$ & $\overline{33}$ & $\overline{11}$ & $\overline{39}$ & \\
\hline $\mathrm{Kt} / \mathrm{V}=\mathrm{I}, 2$ & 4 & 16 & 21 & 84 & 1,00 & 8 & 67 & 17 & 61 & 1,00 \\
\hline $\mathrm{Ht}<33$ & 2 & 15 & II & 85 & & 4 & 33 & 9 & 32 & \\
\hline $\mathrm{Ht}=33$ & 4 & 15 & 23 & 85 & 1,00 & 8 & 67 & 19 & 68 & 1,00 \\
\hline Alb $<3,6$ & 2 & 17 & 10 & 83 & & 7 & 58 & 5 & 18 & \\
\hline$A l b=3,6$ & 4 & 14 & 24 & 85 & 1,00 & 5 & 42 & 23 & 82 & 0,021 \\
\hline
\end{tabular}

* Teste Exato de Fisher

A presença de comorbidades é reconhecida, há vários anos, como a mais importante determinante de sobrevida e morbidade em

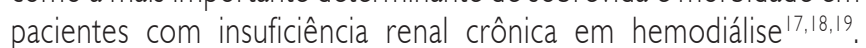
Craven et al. (199|), ao estudarem as comorbidades de pacientes em hemodiálise, usando o IGDR, observaram que os pacientes que foram a óbito em seis meses de seguimento tinham escores médios de 18, enquanto os sobreviventes tiveram escores médios de nove. Aproximadamente 33\% de nossos pacientes apresentaram escores de IGDR superiores a 3I, caracterizando uma população de alta morbidade. De fato, a média dos escores de IGDR dos pacientes que foram a óbito foi significativamente maior que a média dos escores dos sobreviventes $(p<000$ I). Esse mesmo escore também foi maior nos pacientes diabéticos $(p<0,00 \mathrm{I})$. A razão de chances de mortalidade, em relação ao IGDR, foi de I, I0 (IC 95\% : I,02 - I, I8) para cada ponto acrescido no IGDR, comprovando a relevância do instrumento para predizer mortalidade neste grupo de pacientes.

O tempo em tratamento dialítico tem sido descrito como fator associado a maior morbidade 3 . Resultados semelhantes foram encontrados em nosso estudo, uma vez que os pacientes com menos tempo de tratamento, abaixo de um ano, apresentaram tendência a um IGDR menor ( $p=0,060)$. Estes resultados sugerem que o tempo de doença é importante no agravamento das comorbidades, o que é esperado para doenças crônicas. Embora os diabéticos deste estudo fossem em número reduzido, já apresentaram uma tendência a maior risco de mortalidade $(p=0,055)$.

O IGDR, avaliado independentemente do fator diabetes mellitus, apresentou maior preditividade para o evento óbito. No entanto, os pacientes diabéticos tiveram maior IGDR, o que pode ser explicado pelas inúmeras comorbidades destes pacientes, especialmente as cardiovasculares. Numerosos estudos têm associado os indicadores de adequação dialítica à mortalidade de pacientes em hemodiálise $\mathrm{f}^{7,8,9,20,21}$. Diferentemente desses autores, neste estudo não houve associação entre a adequação dialítica e mortalidade. Talvez esta discrepância decorra do tempo de seguimento destes pacientes.

Existem evidências, na literatura, mostrando que os pacientes em hemodiálise apresentam uma melhora na sobrevida, quando um hematócrito normal é atingido ${ }^{23,24}$. Em nosso estudo, não houve associação do hematócrito < 33\% com mortalidade. Estes dados podem ser atribuídos aos bons resultados no controle da anemia na maioria de nossos pacientes, já que 68\% apresentaram níveis de hematócrito superiores a 33\%.
A associação entre desnutrição e mortalidade em pacientes em hemodiálise tem sido descrita por inúmeros pesquisadores ${ }^{12,13,14,22}$. Índices de comorbidade elevados também têm sido relacionados inversamente a níveis baixos de albumina ${ }^{19,26}$. Em nosso estudo, não houve associação estatisticamente significativa entre os níveis de albumina e mortalidade. No entanto, é importante salientar que a maioria dos pacientes com albumina reduzida era de diabéticos. Nessa população, foi observada uma maior mortalidade, quando comparadas aos pacientes não diabéticos, $58 \%$ diabéticos vs $18 \%$ não diabéticos $(p=0,02 \mathrm{I})$. Nesse caso, a redução da albumina deve ter contribuído significativamente para uma maior mortalidade entre os pacientes diabéticos.

\section{ConClusão}

Em nosso estudo, as doenças cardiovasculares foram as que mais contribuíram para compor o IGDR. Observou-se, paralelamente a isso, uma correlação negativa entre albumina e IGDR ( $r=-0,475$; $p=0,002$ ), o que torna possível uma associação entre o marcador nutricional, gravidade da doença renal e mortalidade. Concluindo, os resultados obtidos neste estudo sugerem que o IGDR é um bom instrumento para avaliação do estado de comorbidade de pacientes em hemodiálise, sendo um indicador confiável para comparar grupos de pacientes e predizer mortalidade.

\section{Conflito de interesse: não há.}

\section{SUMMARY}

END-STAGE RENAL DISEASE SEVERITY INDEX, CLINICAL INDICATORS AND MORTALITY OF HEMODIALYSIS PATIENTS

OBJECTIVE. Comorbidity is a major factor influencing mortality in hemodialysis patients. KtN, hematocrit and albumin levels have also been associated with mortality in these patients. The purpose of this study was to evaluate the severity of comorbidity, KtN, hematocritand albumin levels as predictors of mortality in patients on hemodialysis therapy.

METHODS. Forty patients were followed up during 12 months and assessed in relation to social demographic characteristics, time on dialysis therapy, presence of diabetes, KtN, hematocrit and albumin levels, also comorbidities. The impact of comorbidity on mortality was assessed by the end-stage renal disease severity index (ESRD-SI).

RESULTS. Mean ESRD-SI scores for survivals (85\%) and deaths (15\%) were $22 \pm 14.8$ vs. $44 \pm 12.4(p<0.001)$, and fordiabetic (29\%)and non- 
Morsch C et al.

diabetic patients (71\%), 40 15.1 vs. $19 \pm 12.5$ ( $p<0.001$ ). An inverse correlation was observed between ESRD-SI scores and albumin $(r=-$ $0.475 ; p<0.005)$. Albuminlevels $=3.6 \mathrm{~g} / \mathrm{dL}$ were mostly observed $(82 \%)$ in patients without diabetes $(p=0.021)$. A correlation was observed between hematocritand albumin levels $(r=0.544 ; p<0.00$ I). Foreach Ipoint increase in the ESRD-SI scores, there was a $10 \%$ increase in the risk of death $(p=0.0093)$.

CONCLUSION. The ESRD-SI is useful to assess the severity of comorbidities and to predict mortality in hemodialysis patients. [Rev Assoc Med Bras 2005; 5 I(5): 296-300]

KEY wORDS: Hemodialysis. Morbidity. Mortality. Diabetes Mellitus.

\section{REFERÊNCIAS}

I. Amin NS. Assigning severity indices to outcomes. In: Henderson LW, Thuma RS. Quality assurance in dialysis. $2^{\text {nd }}$ ed. Dordrecht: Kluwer Academic Publishers; 1999. p.55-72.

2. Ifudu $O$, Paul HR, Homel P, Friedman EA. Predictive value of functional status for mortality in patients on maintenance hemodialysis. Am J Nephrol 1998; 1 8(2): 109- 16.

3. Miskulin DC, Athienites NV, Yan G, Martin AA, Ornt DB, Kusek JW, Meyer KB, Levey AS. Hemodialysis (HEMO) Study Group. Comorbidity assessment using the Index of coexistent diseases in a multicenter clinical trial. Kidney Int 200 I ;60(4): I 498-5 I0.

4. Craven J, Littlefield C, Rodin G, Murray M. The end-stage renal disease severity index (ESRD-SI). Psychol Med I 99 I;2 I ( I):237-43.

5. Griffin KW, Friend R, Wadhawa NK. Measuring disease severity in patients with end-stage renal disease: validity of the Craven et al. ESRD Severity Index. Psychol Med 1995;25(I): I 89-93.

6. Collins AJ, Ma JZ, Umen A, Keshaviah P. Urea index and other predictors of hemodialysis patient survival. Am J Kidney Dis 1994;24(I): I 57.

7. Hakim RM, Breyer J, Ismail N, Schulman G. Effects of dose of dialysis on morbidity and mortality. Am J Kidney Dis 1994;23(5):66I-9.

8. Held PJ, Port FK, Wolfe RA, Stannard DC, Carroll CE, Daugirdas JT, et al. The dose of hemodialysis and patient mortality. Kidney Int 1996;50(2):550-6.

9. McClellan WM, Soucie JM, Flanders WD. Mortality in end-stage renal disease is associated with facility-to-facility differences in adequacy of hemodialysis. J Am Soc Nephrol 1998;9( I0): 1940-7.

10. Eckardt KU. Target hemoglobin in patients with renal failure. Nephron 2001;89(I): 135-44.

I I. Ifudu $\bigcirc$. Evidence that adequacy of dialisys modulates uremic anemia. Nephron $2001 ; 88(1): 1-5$

12. Bergström J, Lindholm B. Malnutrition, cardiac disease and mortality: an integrated point of view [Editorial review]. Am J Kidney Dis 1998; 32(5):834-4I.
13. Leavey SF, Strawderman RL, Jones CA, Port FK, Held PJ. Simple nutritional indicators as independent predictors of mortality in hemodialysis patients. Am J Kidney Dis 1998;3 I (6):997- I 006.

14. Ikizler TA, Wingard RL, Harvell J, Shyr Y, Hakim RM. Association of morbidity with markers of nutrition and inflammation in chronic hemodialysis patients: a prospective study. Kidney Int 1999;55(I2): | 945-5|.

15. NKF-K/DOQI. Clinical practice guidelines. Am J Kidney Dis 2001;37 (Suppl I):S7-S238

16. Cameron JS. European best practice guidelines for the management of anaemia in patients with chronic renal failure. Nephrol Dial Transplant 1999; | 4(Suppl.2):6 |-5.

17. Locatelli F, Vecchio LD, Manzoni C. Morbidity and mortality on maintenance haemodialysis. Nephron 1998;80(3):380-400.

18. Beddhu S, Bruns FJ, Saul M, Seddon P, Zeidel ML. A simple comorbidity scale predicts clinical outcomes and costs in dialysis patients. Am J Med 2000; 108(8):609- 13

19. Davies SJ, Phillips L, Naish PF, Russell Gl. Quantifying comorbidity in peritoneal dialysis patients and its relationship to other predictors of survival. Nephrol Dial Transplant 2002; | 7( | 0): I 085-92.

20. Himmelfarb J, Holbrook D, McMonagle E, Robinson R, Nye L, Spratt D. $\mathrm{Kt} / \mathrm{N}$, nutritional parameters, serum cortisol, and insulin growth factor- I levels and patient outcome in hemodialysis. Am J Kidney Dis 1994; 24(4):473-9.

2 I. Parker TF, Husni L, Huang W, Lew N, Lowrie EG. Survival of hemodialysis patients in the United States is improved with a greater quantity of dialysis. Am J Kidney Dis 1994;23(5):670-80.

22. Chertow GM, Owen WF, Lazarus JM, Lew NL, Lowrie EG. Exploring the reverse J-shaped curve between urea reduction ratio and mortality. Kidney Int 1998;56( I I): I 872-8.

23. Valderrábano F, Jofre R, López-Gómez J. Quality of life in end-stage renal disease patients. Am J Kidney Dis 2001 ;38(3):443-64.

24. Donne RL, Foley RN. Anaemia management and cardiomyopathy in renal failure. Nephrol Dial Transplant 2002; I 7(Suppl I):37-40.

25. Chertow G. Modality-specific nutrition support in ESRD: weighing the evidence. Am J Kidney Dis 1999;33( I): 193-7.

26. Zimmermann J, Herrlinger S, Pruy A, Metzger T, Wanner C. Inflammation enhances cardiovascular risk and mortality in haemodialysis patients. Kidney Int 1999;55(6):648-58.

Artigo recebido: 21/06/04 Aceito para publicação: 03/06/05 